\title{
Adaptation, acceptability and feasibility of a Short Food Survey to assess the dietary intake of children during attendance at childcare
}

\author{
Alice Grady 1,2,3,4,* (1), Alison Fielding ${ }^{1,5}$, Rebecca K Golley6, Meghan Finch 1,4,7, \\ Gilly A Hendrie ${ }^{8}$, Tracy Burrows ${ }^{7,9}$, Kirsty Seward ${ }^{1,2,3,4}$, Christophe Lecathelinais ${ }^{2}$ and \\ Sze Lin Yoong 1,2,3,4 \\ 'School of Medicine and Public Health, University of Newcastle, Callaghan, NSW, Australia: ${ }^{2}$ Hunter New England \\ Local Health District, Population Health, Wallsend, NSW, Australia: ${ }^{3}$ Hunter Medical Research Institute, Newcastle, \\ NSW, Australia: ${ }^{4}$ Priority Research Centre for Health Behaviour, University of Newcastle, Callaghan, NSW, Australia: \\ ${ }^{5}$ GP Synergy, Research and Evaluation Unit, Mayfield West, NSW, Australia: ${ }^{6}$ College of Nursing and Health \\ Sciences, Flinders University, Adelaide, SA, Australia: ${ }^{7}$ Priority Research Centre in Physical Activity and Nutrition, \\ University of Newcastle, Callaghan, NSW, Australia: ${ }^{8}$ Health and Biosecurity, Commonwealth Scientific and \\ Industrial Research Organisation, Adelaide, SA, Australia: ${ }^{9}$ School of Health Sciences, Faculty of Health and \\ Medicine, University of Newcastle, Callaghan, NSW, Australia
}

Submitted 17 June 2019: Final revision received 5 September 2019: Accepted 17 September 2019: First published online 17 March 2020

\begin{abstract}
Objective: To (i) describe the adaptation of the Short Food Survey (SFS) for assessing the dietary intake of children (2-5 years) during attendance at Early Childhood Education and Care (SFS-ECEC); (ii) determine the acceptability and feasibility of the SFS-ECEC; and (iii) compare the SFS-ECEC to direct observations for assessing dietary intake of children in care.

Design: The adapted forty-seven-item SFS-ECEC was completed by childcare educators to capture individual child's usual intake over the past month. Acceptability and feasibility were assessed via educator self-report and completion rates. Mean servings of food groups consumed in accordance with dietary guidelines reported in the SFS-ECEC were compared to those obtained by a single-day direct observation via visual estimation conducted by trained personnel. Mean differences, intra-class correlations, Bland-Altman plots, percentage agreement and Cohen's $\kappa$ were examined.

Setting: Early Childhood Education and Care, NSW, Australia.

Participants: Educators and children.

Results: 213 (98.61\%) SFS-ECECs were returned. Acceptability was high with $86.54 \%$ of educators reporting the tool as easy to understand. Mean differences in servings of food groups between the SFS-ECEC and direct observation were statistically significantly different for five out of six foods and ranged 0.08-1.07, with intra-class correlations ranging $0 \cdot 00-0 \cdot 21$. Agreement between the methods in the classification of children meeting or not meeting dietary guidelines ranged 42.78-93.01\%, with Cohen's $\kappa$ ranging -0.03 to $0 \cdot 14$.

Conclusions: The SFS-ECEC is acceptable and feasible for completion by childcare educators. While tool refinement and further validation is warranted, small mean differences suggest the tool may be useful in estimating group-level intakes.
\end{abstract}

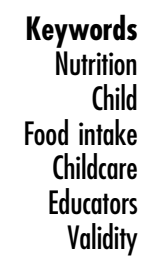

Early childhood is a critical period for establishing dietary intake patterns for lifelong health and is a key life-stage for obesity prevention initiatives ${ }^{(1-3)}$. Early Childhood Education and Care (ECEC) services are gaining increasing recognition as a key setting for nutrition-related primary prevention initiatives ${ }^{(4)}$ with growing childcare service utilisation globally ${ }^{(3,5-8)}$. Feasible, accurate and reliable dietary intake measures are essential for assessing the

*Corresponding author: Email Alice.Grady@health.nsw.gov.au

(C) The Authors 2020. This is an Open Access article, distributed under the terms of the Creative Commons Attribution licence (http:// creativecommons.org/licenses/by/4.0/), which permits unrestricted reuse, distribution, and reproduction in any medium, provided the original work is properly cited. 
effectiveness of interventions aimed at improving children's intake in the care setting, as well as for monitoring adherence to childcare-specific dietary guidelines ${ }^{(2,3,6,9)}$.

Measuring dietary intake in young children is complex, where the usual challenges of dietary assessments are compounded by the inability of young children to self-report due to limited communication, knowledge of food types and cognitive functioning ${ }^{(9,10)}$. Parents/guardians are, therefore, often required to act as proxy-reporters, yet their ability to accurately report food consumed is inherently limited for the meals and snacks provided during childcare $\operatorname{hours}^{(9,10)}$

The assessment of dietary intake of children in care settings, to date, has been largely dependent on direct observations or objective data collection of food consumption (e.g. plate-waste measures) ${ }^{(5)}$. While direct observation is considered valid and reliable, ${ }^{(11)}$ this method is costly to conduct, limiting its feasibility as a method for assessing the dietary intake of young children in childcare on a population level ${ }^{(3,9)}$. As such, the practicality of direct observation for epidemiological or evaluation research involving large numbers of children in care settings dispersed across wide geographical areas is limited.

Alternatives to direct observation and collection of objective data exist. There is rapid development in the utilisation of technology-based methods (e.g. photo- or videorecordings) for measuring the dietary intake of children ${ }^{(2,9)}$. However, such methods may be prohibitive due to pragmatic issues, including consent and privacy concerns for young children and childcare services, in addition to costs associated with training staff in the use of technologies, ongoing development and validity concerns. One nonvalidated educator-reported (as proxy reporters) method of assessing individual child's intake in care has also been reported in the literature ${ }^{(12,13)}$. However, as this method requires detailed real-time recordings of intake by educators, feasibility limitations are apparent given the burden on time-limited childcare educators and potential impact on service daily schedules and routines.

Short survey-style tools completed by childcare educators provide a potential solution to the challenges faced in measuring individual children's dietary intake at childcare. Short tools tend to be less than fifty items in length and ask questions about the frequency or servings of foods or food groups, as well as diet quality or dietary habits ${ }^{(1,14)}$. They are quick to administer, with low respondent and administrator burden, and are therefore more feasible for the assessment of dietary intake of children at scale. Reviews of short tools that measure children's dietary intake have highlighted a lack of validated short dietary intake methods to assess 'in-care' intake of children ${ }^{(1)}$.

One short survey tool identified in a recent review with favourable validity and reliability for assessing the dietary intake of young children, compared with other short food questionnaires, is the Short Food Survey (SFS) ${ }^{(14)}$. The SFS is a validated, thirty-eight-item parent-reported measure of individual child's dietary intake, developed for the Australian population ${ }^{(15)}$. It has been found to perform well in estimating intake across a range of food groups in children aged 4-11 years and for estimating children's overall adherence to the Australian Dietary Guidelines via an index score ${ }^{(15)}$. On this basis, the SFS has potential utility to be adapted for completion by childcare educators, where maximising accuracy while also maintaining educator acceptability and feasibility are paramount considerations.

Therefore, the aims of this study were to (i) describe the adaptation of the SFS for assessing dietary intake of children (aged 2-5 years) during attendance at ECEC services; (ii) determine the acceptability and feasibility of the SFSECEC as reported by a sample of childcare educators; and (iii) compare the SFS-ECEC to direct observations for assessing the daily intake of core food groups (fruit, vegetables, breads and cereals, meat/meat alternatives, dairy/ dairy alternatives) and discretionary foods, according to the Australian Guide to Healthy Eating (AGHE), of children attending childcare.

\section{Methods}

\section{Adaptation of the Short Food Survey for use in Early Childbood Education and Care}

An advisory group consisting of dietitians, public health nutritionists, health promotion officers with expertise in the ECEC setting, and the developers of the SFS was formed to identify modifications required to the original SFS for application in the childcare setting using an iterative adaptation and review process (online supplementary material, Supplemental Appendix A for details of adaptations made). In brief, this encompassed modifications to food items assessed/food item examples based on relevance to the ECEC setting; modification of frequency response options to accommodate the assessment of children that attend care for varying numbers of days per week; paper-based survey administration; the addition of portion-size questions to enable the estimation of food group servings; and development of a twelve-page supporting resource flipchart containing images and written examples of serving size portions to assist educators in estimating the food intake of children and categorising foods into groups.

The above process resulted in a forty-seven-item educator-completed dietary assessment tool to capture information on individual child's (aged 2-5 years) food group intakes and food choices in care over the past month (online supplementary material, Supplemental Appendix B for SFS-ECEC items and responses). Intake and food choice assessment covered the days of the week the child usually attended the childcare service, and the meals the child usually consumed while in care, including breakfast, morning tea, lunch, afternoon tea and late snack. Food groups included the five core food groups described 
in the $\mathrm{AGHE}^{(16)}$, which are fruit, vegetables, breads and cereals, meat/meat alternatives and dairy/dairy alternatives, as well as discretionary choices (i.e. food or beverage items high in saturated fat, added sugars or sodium, and may be energy-dense). The tool also asked about food behaviours, including the consumption of wholegrain choices, reduced-fat dairy, healthy fats and variety (of choices within and between core food groups) ${ }^{(15)}$.

The SFS-ECEC captured data on:

1. Days of the week the child attends care and meals consumed (two items)

2. Frequency of consumption of core AGHE food group items and water (eighteen items)

3. Portion sizes consumed for core AGHE food group items (ten items)

4. Frequency of consumption of discretionary food/ beverage items (ten items)

5. Food variety (types of fruit, vegetables, dairy/dairy alternatives, meat/meat alternatives and breads and cereals) (three items)

6. Food choices (wholegrains, fat content of milk, trimmed meat, spread type) (four items)

Pilot-testing of the initial version of the SFS-ECEC was undertaken with a convenience sample of four educators from two childcare services (not included in the current study). Educators were provided verbal and written instructions for the pilot SFS-ECEC and were asked to complete the tool for at least one child each. Feedback was sought, covering tool length, comprehension and feasibility of completion. Feedback supported the feasibility and acceptability of the initial version.

\section{Acceptability, feasibility and comparison of the Short Food Survey-Early Childhood Education and Care to direct observations}

\section{Design and setting}

An opportunistic cross-sectional study was undertaken to assess SFS-ECEC acceptability, feasibility and to compare two measures of dietary intake of children attending care. The study took place as part of baseline data collection within a randomly selected subset of ECEC services taking part in a randomised controlled trial aiming to improve childcare service compliance with dietary guidelines ${ }^{(7,17)}$.

\section{Sample}

ECEC services in New South Wales (NSW), Australia, who were clients of a single, specific childcare management software provider, were invited to participate in the randomised controlled trial. To be eligible for this sub-study, services were required to: (i) be open for $\geq 8 \mathrm{~h}$ each weekday; (ii) prepare and provide at least one main meal and two snacks daily to children on-site; and (iii) be able to make menu-planning decisions on-site; and be randomly selected to participate in a 1-day site visit from the research team for the purposes of data collection. Services outsourcing menu planning, not catering for children aged 3-6 years, catering exclusively for special needs children or run by the Department of Education and Communities were excluded.

Educators were required to be present on the day of data collection and allocated to the room in which the highest number of children aged 2-5 years were located. This age range was selected to assess individual child's intake in care against dietary guidelines for the sector, Caring for Children ${ }^{(17)}$. A pragmatic approach to selecting educators was used to maximise the feasibility of completing individual SFS-ECEC records. Participating educators were required to have familiarity with the typical dietary intake of children undergoing direct observation, as indicated by working regularly (preferably permanently) in the selected room and not being new to the service or room in which dietary observations were taking place.

\section{Child recruitment}

Services provided information statements and consent forms to the parents of potentially eligible children approximately 2 weeks prior to the site visit day. Research assistants present on the day of data collection also approached the parents/guardians during child drop-off to assess eligibility and obtain informed consent for their child to take part.

Children aged 2-5 years, present on the day of data collection, with parent/guardian consent, and with no dietary requirements prohibiting the consumption of foods usually provided were included in the study.

\section{Data collection procedures}

Direct observations (reference method). Children's dietary intake was measured by research assistants in six randomly selected children per service. Random selection was conducted by a blinded research assistant on the morning of the observation. All participating children were assigned a unique identification number, which was matched against a computer-generated random number table to select the children to be observed. Observations were undertaken using an adapted protocol of the Dietary Observation for Child Care (DOCC) ${ }^{(18)}$, a validated approach for assessing dietary intake in children aged $3-5$ years. The DOCC is considered sound for the assessment of dietary intake of children in care ${ }^{(11)}$. As detailed in the protocol for the larger randomised controlled trial ${ }^{(7)}$, prior to service site visits, the research assistants undertook laboratory-based training to ensure accurate identification and estimation of portion sizes of foods and beverages commonly consumed by children in the ECEC setting. Two observers each assessed three children on the day, by documenting the types and portion sizes of food and beverages served to each individual child. At the end of each core meal (morning tea, lunch, afternoon 
tea), observers recorded the types and portion sizes of foods remaining on the individual child's plates. Portion sizes were estimated using household measures (e.g. tablespoons), units for foods that are counted in units (e.g. nuggets) or the dimension of foods in centimetres. As per the DOCC protocol, estimated consumption of foods in grams was then calculated ${ }^{(18,19)}$ by a dietitian via Foodworks $9^{(20)}$. Servings of discretionary foods were calculated by dividing the kilojoules consumed from discretionary foods by a standard serving size $(600 \mathrm{~kJ})$ according to $\mathrm{AGHE}^{(16)}$. The core food group data were entered into an online programme developed by the research team to classify the number of servings for each food group according to $\mathrm{AGHE}^{(16)}$.

Administration of the SFS-ECEC. Educators from the room in which the randomly selected children were located completed written questionnaires reporting on the selected children's characteristics and dietary intake via SFS-ECEC. Educators were asked to complete one SFS-ECEC for each randomly selected child and were asked to complete the SFS-ECECs on the day of site visit or, if this was not possible, to return the completed SFS-ECECs to the research team at a later date via reply paid envelopes provided to the service. Prior to SFS-ECEC completion, the research assistants provided all participating educators with brief training on how to accurately complete the survey, and supporting resources containing example images of foods commonly served in childcare to help with estimating portion sizes. Educators were asked to refer to this supporting resource wherever possible. Immediately following the completion of the SFS-ECEC, educators were asked to complete a brief questionnaire assessing their demographic characteristics, and acceptability and feasibility of the tool.

Acceptability and feasibility. Four items were developed by the research team on a four-point Likert scale (ranging from strongly disagree to strongly agree) assessing the clarity ('I found the questionnaire clear') and comprehension ('I found the questionnaire easy to understand') of the tool, adverse consequences of completion ('I found the questionnaire distressing') and usefulness of the supporting resource ('I found the supporting resource useful for completing the questionnaire') to assess acceptability ${ }^{(21)}$. These items were completed immediately following the completion of the SFS-ECEC. The feasibility ${ }^{(21)}$ of the SFS-ECEC was also determined by calculating response rate, frequency of missing items, Flesch-Kincaid reading level, self-reported number of SFS-ECECs completed, time to complete, and timeframe from provision of SFS-ECECs to completion.

Servings of food groups. Educators reported on children's frequency of consumption of all six food groups over the past month (thirty-seven items) within the SFSECEC for the meals and snacks the child usually consumes in care (two items). Core food groups were also reported by amount in portions (e.g. two portions is equivalent to one serving size consistent with AGHE). Usual frequency and
Table 1 Australian Guide to Healthy Eating food groups and recommended servings for children while attending care according to the New South Wales Caring for Children Guidelines ${ }^{(17)}$

\begin{tabular}{lc}
\hline Food group & $\begin{array}{c}\text { Recommended } \\
\text { servings }\end{array}$ \\
\hline Fruit & 1 \\
Vegetables & 2 \\
Wholegrain breads and cereals & 2 \\
$\begin{array}{l}\text { Lean meat and alternatives (e.g. poultry, } \\
\text { fish, eggs, tofu, seeds and legumes) }\end{array}$ & $0 \cdot 75$ \\
$\begin{array}{c}\text { Dairy (e.g. milk, yoghurt, cheese and } \\
\text { non-dairy alternatives) }\end{array}$ & 1 \\
$\begin{array}{c}\text { Discretionary foods (e.g. high in kilojoules, } \\
\text { saturated fat, added sugars and added salt) }\end{array}$ & 0 \\
\hline
\end{tabular}

portions of core food groups consumed were then converted into servings per day. As the SFS-ECEC assessed the number of times discretionary foods were consumed rather than servings, a standardised serving size, calculated from the mean kilojoules each time a discretionary food was consumed from direct observations $(626 \mathrm{~kJ})$, was applied to the discretionary food frequency of consumption data. As per direct observations, the servings of discretionary foods were then calculated by dividing the kilojoules consumed from discretionary foods by the standard serving size $(600 \mathrm{~kJ})$ according to $\mathrm{AGHE}^{(16)}$.

Compliance with childcare guidelines. According to the NSW dietary guidelines for the sector ${ }^{(17)}$, childcare services are required to provide at least one main meal (e.g. lunch) and two mid-meals or snacks (e.g. morning tea and afternoon tea) over an 8 -h period, with these meals providing at least $50 \%$ of the recommended daily servings of each of the AGHE food groups (Table 1). Each food group was considered compliant when the recommended number of servings for that food group according to the NSW dietary guidelines for the sector ${ }^{(17)}$ was observed or reported to be consumed. For direct observations, this reflected the consumption of recommended servings of all food groups on the day of observation; for the SFSECEC, this reflected usual daily consumption of recommended servings reported by educators for each child over the past month.

\section{Other data}

Childcare service characteristics, including postcode, number of allocated places and number of educators, were reported by the service-nominated supervisor during service recruitment. Educator characteristics, including qualifications, years worked for the services and days worked each week, and child's characteristics, including age, sex, days usually attending care and meals and snacks usually consumed in care, were reported by educators.

\section{Statistical analysis}

Statistical analyses were conducted in SAS, version $9.3^{(22)}$. Descriptive statistics, including means, frequencies and 
proportions, were used to describe the characteristics of childcare services, educators and children, consumption servings of food groups and compliance with guidelines. Service postcodes, ranked in the bottom $50 \%$ of NSW according to the Socio-Economic Indexes for Areas $2016^{(23)}$, were classified as being of lower socioeconomic status. Items within the SFS-ECEC without a response were recorded as missing. The following exclusions were made to the dataset: direct observations of children without a corresponding SFS-ECEC and late completers of SFS-ECEC as identified by return of surveys over 1 month post-direct observations. This resulted in a total of two services (encompassing two educators) and twenty-one children ( $9.72 \%$ ) being excluded from the analyses. Further, outliers as identified by food group servings $>3$ SD from the mean intake according to either direct observations or SFS-ECEC were removed from individual food group analyses (ranging 1-9 per food group).

Multiple methods were employed to compare the estimates of intake derived from the SFS-ECEC to direct observations. For servings of food groups (continuous data), differences between the two methods in mean estimates of intake were assessed using linear mixed model regression with the childcare services as a random effect to account for potential clustering effect. Intra-class correlations (ICC) were calculated to establish group-level association between the two methods ${ }^{(24)}$; Bland-Altman plots to determine the agreement between individual's absolute values from each method ${ }^{(25)}$; and linear regression analysis for each food group (regression of the average of two methods $v$. their difference) to test if the slope of mean bias was significantly different from zero (group level) $^{(25)}$. Visual examination of histograms determined the mean difference in servings for each food group to be normally distributed. The ability of the SFS-ECEC to categorise children into meeting/not meeting dietary consumption recommendations for each food group (dichotomous data) was also assessed using percentage perfect agreement ${ }^{(15)}$; McNemar's test to determine the significance of differences between SFS-ECECs and direct observations; and Cohen's $\kappa^{(15)}$. Based on the benchmarks suggested by Landis and $\operatorname{Koch}^{(26)}, \kappa$ measures were classified as follows: poor $=<0$; slight $=0 \cdot 00-0 \cdot 20$; fair $=$ $0 \cdot 21-0.40 ; \quad$ moderate $=0 \cdot 41-0 \cdot 60 ; \quad$ substantial $=0 \cdot 61-0 \cdot 80$; almost perfect $=0 \cdot 81-1 \cdot 00$.

For the comparison of mean servings of food groups consumed, a total of 195 children across 33 services, assuming an ICC of 0.05 and with an $\alpha$ of 0.05 , had $80 \%$ power to detect a difference of $0.32 \mathrm{Z}$ units (or $32 \%$ of an SD) between the SFS-ECEC and direct observations.

\section{Results}

A total of 33 services, 52 educators and 195 children were included in the analyses (Table 2). On average, educators
Table 2 Characteristics of participating childcare services ( $n 33$ ), educators ( $n$ 52) and children ( $n$ 195)

\begin{tabular}{|c|c|c|}
\hline Characteristics & $n$ or mean & $\%$ or SD \\
\hline \multicolumn{3}{|l|}{ Service } \\
\hline \multicolumn{3}{|l|}{ Socioeconomic status } \\
\hline High & 20 & $60 \cdot 61$ \\
\hline Low & 13 & 39.39 \\
\hline $\begin{array}{l}\text { Mean number of allocated } \\
\text { childcare places per day }\end{array}$ & $56 \cdot 82$ & $16 \cdot 12$ \\
\hline $\begin{array}{l}\text { Mean number of educators } \\
\text { per service }\end{array}$ & $12 \cdot 58$ & 8.76 \\
\hline \multicolumn{3}{|l|}{ Educator } \\
\hline \multicolumn{3}{|l|}{ Number of years employed } \\
\hline$<1$ year & 15 & $28 \cdot 85$ \\
\hline $1-5$ years & 22 & $42 \cdot 31$ \\
\hline$>5$ years & 15 & $28 \cdot 85$ \\
\hline $\begin{array}{l}\text { Mean number of days employed } \\
\text { at service per week }\end{array}$ & $4 \cdot 75$ & 0.58 \\
\hline \multicolumn{3}{|l|}{ Highest qualification completed } \\
\hline Early childhood teaching & 27 & 51.92 \\
\hline Diploma & 16 & 30.77 \\
\hline Certificate III & 7 & 13.46 \\
\hline Other & 2 & 3.85 \\
\hline \multicolumn{3}{|l|}{ Child } \\
\hline Mean age & 4.09 & 0.71 \\
\hline \multicolumn{3}{|l|}{ Sex } \\
\hline Female & 91 & $48 \cdot 40$ \\
\hline Male & 97 & $51 \cdot 60$ \\
\hline $\begin{array}{l}\text { Mean number of days in } \\
\text { care per week }\end{array}$ & 3.42 & $1 \cdot 15$ \\
\hline \multicolumn{3}{|l|}{ Meals consumed while in care } \\
\hline Breakfast & 29 & $15 \cdot 18$ \\
\hline Morning tea & 173 & 90.58 \\
\hline Lunch & 190 & $99 \cdot 48$ \\
\hline Afternoon tea & 190 & $99 \cdot 48$ \\
\hline Late snack & 49 & $25 \cdot 65$ \\
\hline
\end{tabular}

were present for almost $5 \mathrm{~d}$ of the week, children attended the service $3.42 \mathrm{~d}$ out of 5 , and over $90 \%$ of children regularly consumed morning tea, lunch and afternoon tea in care, with an additional $15 \cdot 18 \%$ of children consuming breakfast and $25.65 \%$ consuming a late snack.

\section{Acceptability, feasibility and comparison of the Short Food Survey-Early Childhood Education and Care to direct observations}

The acceptability assessment of the SFS-ECEC determined that $86.54 \%$ of educators agreed that they found the questionnaire clear, $86.54 \%$ agreed that they found the questionnaire easy to understand, $96 \cdot 15 \%$ found the supporting resource useful for completing the questionnaire, and $19.61 \%$ reported they found the questionnaire distressing. Difficulties in accurately capturing children's consumption due to variation in child and staff attendance, child self-serving and changing menu cycles were reported by five educators.

Of the 216 SFS-ECECs distributed to educators, 213 (98.61\%) were returned. The frequency of missing responses of any one item ranged 6-11 (3.08-5.64\%). In terms of readability, the Flesch Reading Ease was 64.7 (plain English, easily understood by 13-15-year-olds), 
Table 3 Comparison of servings per day for core food groups and discretionary foods calculated using Short Food Survey-Early Childhood Education and Care (SFS-ECEC) and direct observations

\begin{tabular}{|c|c|c|c|c|c|c|c|c|c|}
\hline & \multicolumn{2}{|c|}{ SFS-ECEC } & \multicolumn{2}{|c|}{$\begin{array}{c}\text { Direct } \\
\text { observations }\end{array}$} & \multirow[b]{2}{*}{ Mean difference } & \multirow[b]{2}{*}{$95 \% \mathrm{Cl}^{\mathrm{a}}$} & \multirow[b]{2}{*}{ ICC } & \multirow[b]{2}{*}{$95 \% \mathrm{Cl}$} & \multirow[b]{2}{*}{ Slope of bias ${ }^{b}$} \\
\hline & Mean & SD & Mean & SD & & & & & \\
\hline Fruit & 0.78 & 0.48 & 0.70 & 0.72 & 0.08 & $-0.03,0.20$ & 0.00 & - & $-0.72^{\star \star}$ \\
\hline Vegetables & 0.78 & 0.50 & 0.59 & 0.53 & 0.19 & $0.10,0.29^{\star \star}$ & 0.11 & $0.03,0.34$ & -0.11 \\
\hline Breads and cereals & 2.41 & 1.75 & 1.35 & 0.82 & 1.07 & $0.83,1.32^{\star *}$ & 0.07 & $0.01,0.42$ & $1 \cdot 14^{\star *}$ \\
\hline Dairy/dairy alternatives & 1.22 & 0.75 & 0.74 & 0.63 & 0.48 & $0.35,0.61^{\star \star}$ & 0.14 & $0.05,0.34$ & $0.26^{*}$ \\
\hline Meat/meat alternatives & 0.69 & 0.47 & 0.32 & 0.40 & 0.37 & $0.29,0.45^{\star \star}$ & 0.00 & - & $0.26^{\star}$ \\
\hline Discretionary foods & 0.68 & 0.49 & 0.41 & 0.71 & 0.28 & $0.18,0.39^{\star *}$ & 0.21 & $0.11,0.37$ & $-0.55^{\star \star}$ \\
\hline
\end{tabular}

aLinear mixed models regression with random effects accounting for clustering.

bLinear regression analysis (regression of the average of two methods $v$. their difference).

${ }^{\star} P<0.05 ;{ }^{\star \star} P<0.001$.

and the Flesch-Kincaid Grade Level was 7.9. On average, educators completed 3.61 (SD 1.81) SFS-ECECs, taking an average of 13.59 (SD 8.37) min to complete per child. The questionnaires were completed on average $3 \mathrm{~d}$ following direct observation data collection.

\section{Servings of food groups}

The mean difference in the servings of each core food group and discretionary foods between the two measures ranged 0.08-1.07 servings (Table 3), with the SFS-ECEC reporting relatively higher consumption compared to direct observation. Significant differences were found in the mean servings of vegetables, breads and cereals, dairy/ dairy alternatives, meat/meat alternatives and discretionary foods between the two methods. The ICC for food group servings was lowest for fruit and meat/meat alternatives (ICC $=0.00)$. A negative slope of bias was found for three of the six food groups, with linear regression analysis revealing the slope of bias was significantly different from zero for all food groups, with the exception of vegetables.

A visual examination of Bland-Altman plots (Fig. 1) revealed that with regard to measures of fruit, as the mean of the two measures increased, the greater the underestimation of SFS-ECEC compared to direct observations. For breads and cereals and meat/meat alternatives, as the mean of the two measures increased, the greater the overestimation of SFS-ECEC compared to direct observations; and for vegetables, dairy/dairy alternatives and discretionary foods, as the mean of the two measures increased, the greater the dispersion of SFS-ECEC compared to direct observations.

\section{Compliance with guidelines}

The proportion of children meeting dietary guidelines for the sector while attending care according to the SFSECEC ranged 4.76-57.29\%, with discretionary foods showing lowest compliance and dairy/dairy alternatives showing highest compliance (Table 4). Compliance via direct observations ranged 2.08-61.03\%, with lowest compliance for vegetables and highest compliance for discretionary foods. Percentage agreement between SFS-ECEC and direct observations ranged $42.78-93.01 \%$ across all food groups, with significant differences in classifications of meeting or not meeting for all food groups, apart from vegetables $(P=0 \cdot 17) . \kappa$ coefficient values suggest poor agreement between the two measures for fruit and vegetables ( $\kappa$ ranging -0.03 to -0.02$)$ and slight agreement for the remaining food groups ( $\kappa$ ranging $0 \cdot 04-0 \cdot 14$ ). Vegetables had the highest percentage agreement between the two methods, and yet a negative $\kappa$ due to the unequal distribution of agreement between meeting and not meeting guidelines.

\section{Discussion}

This paper describes the adaptation, acceptability and feasibility of the SFS-ECEC and compares the adapted tool to a single day of direct dietary observations. The SFS-ECEC was based on a previously validated, parent-reported tool, which assesses usual food group consumption and overall diet quality in young children ${ }^{(15)}$. A team of dietitians, public health nutritionists, health promotion officers and SFS tool developers made a number of modifications to the original SFS tool, to enable the assessment of dietary intake of children while attending childcare, to be completed by educators.

The study found high acceptability among childcare staff, as the majority of educators (86.54-96.15\%) reported the SFS-ECEC to be clear, easy to understand and almost all found the flipchart resource helpful to support reporting the dietary intake of children. Surprisingly, $19.61 \%$ of educators reported they found the questionnaire distressing. As this was the only negatively worded item, it is suspected that this finding may indicate response acquiesce. It is also suspected that this distress is related to the overall workload experienced by some educators, with the completion of the tool representing an additional, cognitively challenging task to be completed within a short timeframe. The task of reporting the frequency and portion 
(a)

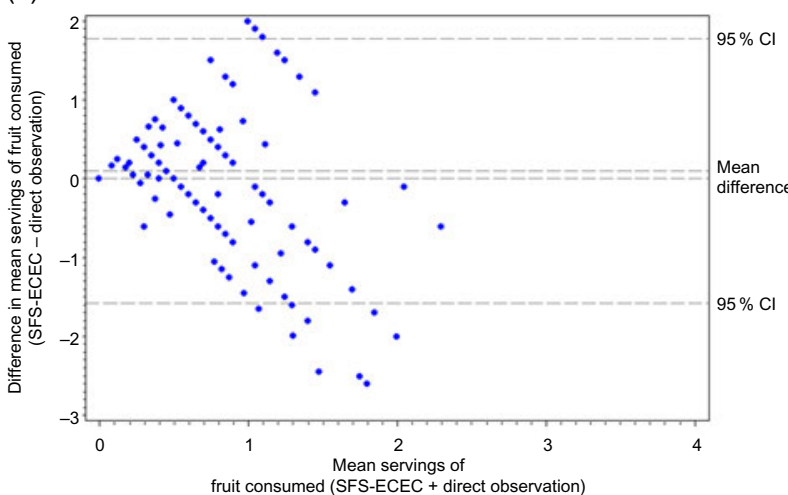

(c)

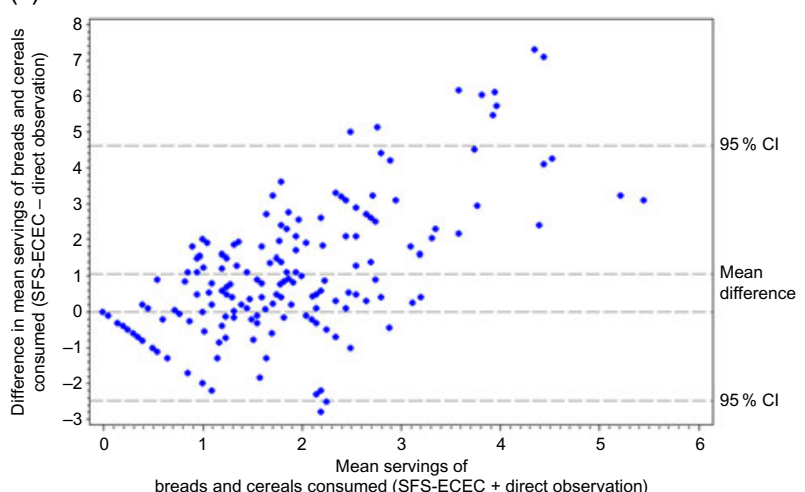

(e)

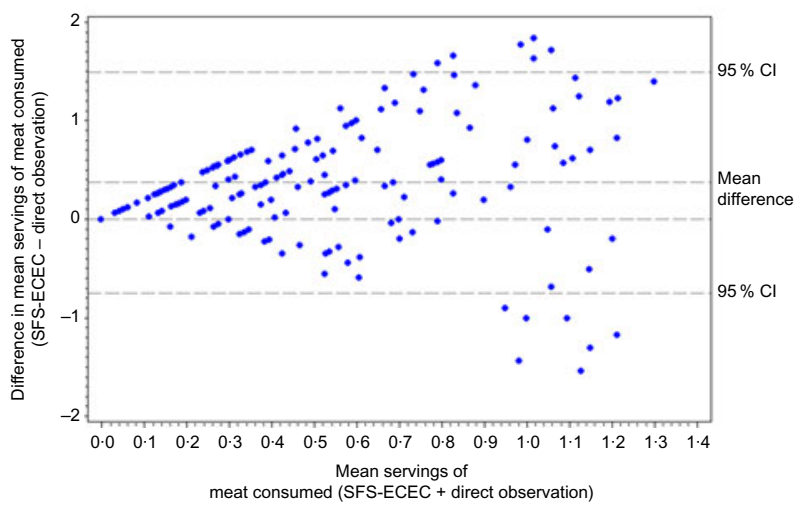

(b)

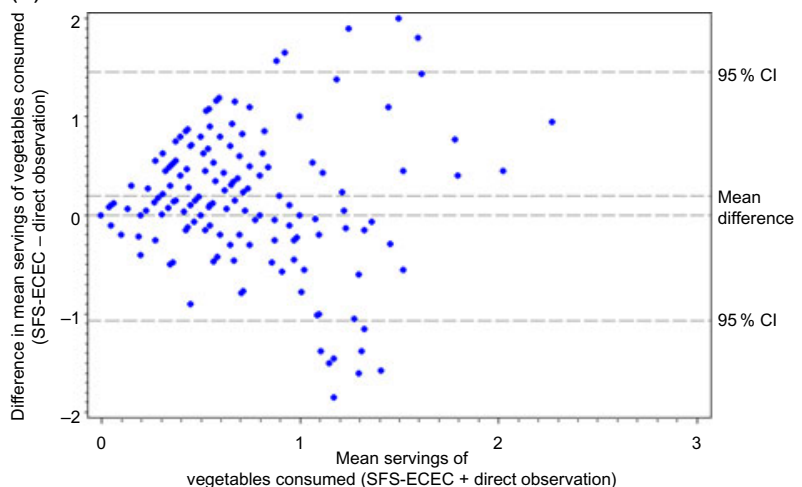

(d)

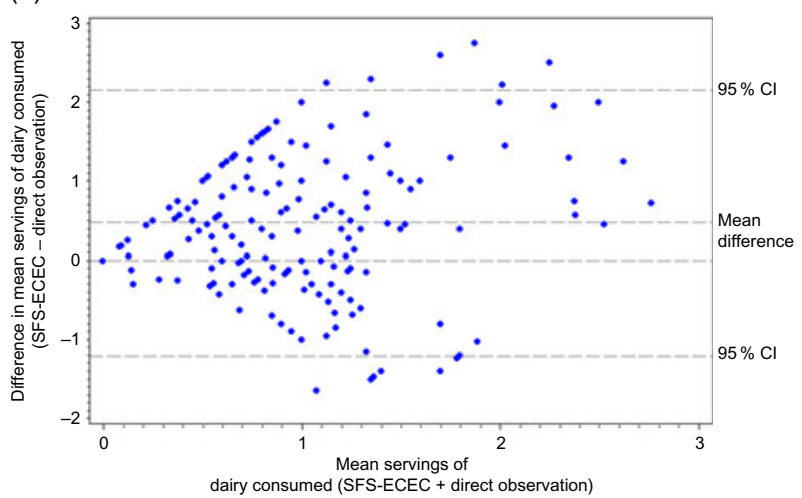

(f)

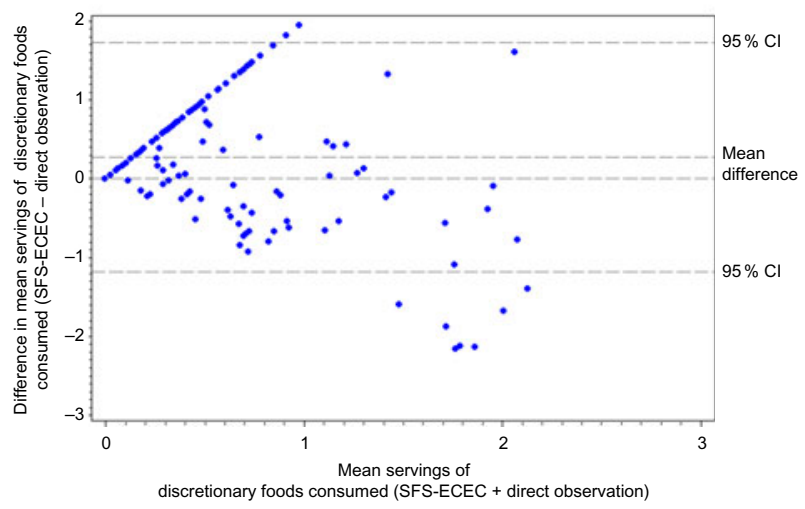

Fig. 1 (colour online) Bland-Altman plots showing agreement between consumption of servings for each food group calculated by Short Food Survey-Early Childhood Education and Care (SFS-ECEC) and direct observations for the following food groups: (a) fruit, (b) vegetables, (c) breads and cereals, (d) dairy/dairy alternatives, (e) meat/meat alternatives and (f) discretionary foods. For each food group, the mean difference in servings between SFS-ECEC and direct observations ( $y$ axis) was plotted against the mean servings calculated by SFS-ECEC and direct observations ( $x$ axis), including mean differences and $95 \% \mathrm{Cls}$

size for individual children may be difficult for educators in services that encourage children to serve themselves from shared plates or 'family style ${ }^{(27)}$. Serving styles, in addition to daily variation in the servings of meals and snacks, the frequency of staff working and the frequency of child attendance per week were noted by five educators as challenges to tool completion. Despite this, the feasibility of tool completion in the childcare setting was high as almost all (98.61\%) of the distributed SFS-ECECs were returned by educators. The average time of completion was $13.6 \mathrm{~min}$ per child, and on average, educators completed 3.6 surveys. This is encouraging when compared to direct observations that are conducted over $6 \mathrm{~h}$, with a maximum of three children per observer. Broadly, such findings are reassuring and suggest that the SFSECEC is an acceptable and feasible tool to be completed by educators; however, follow-up questions surrounding potential adverse consequences may be warranted.

Overall, the SFS-ECEC reported a higher number of servings for all core food groups and discretionary foods relative to direct observations (mean difference ranging 
Table 4 Proportion of children meeting dietary guidelines for the sector, and percentage agreement between SFS-ECEC and direct observations categorised by meeting/not meeting food group recommendations

\begin{tabular}{|c|c|c|c|c|c|c|c|c|}
\hline \multirow[b]{2}{*}{ Food group } & \multicolumn{2}{|c|}{ SFS-ECEC } & \multicolumn{2}{|c|}{$\begin{array}{c}\text { Diet } \\
\text { observations }\end{array}$} & \multirow[b]{2}{*}{$P$-value ${ }^{\mathrm{a}}$} & \multirow[b]{2}{*}{ Percent perfect agreement } & \multirow[b]{2}{*}{ Cohen's $\kappa$} & \multirow[b]{2}{*}{$95 \% \mathrm{Cl}$} \\
\hline & $n$ & $\%$ & $n$ & $\%$ & & & & \\
\hline Fruit & 80 & 41.88 & 45 & 23.08 & $<0.001$ & 53.40 & -0.02 & $-0.15,0.10$ \\
\hline Vegetables & 9 & 4.76 & 4 & 2.08 & 0.17 & 93.01 & -0.03 & $-0.05,-0.01$ \\
\hline Breads and cereals & 107 & $56 \cdot 02$ & 42 & 21.88 & $<0.001$ & 54.26 & 0.14 & $0.03,0.24$ \\
\hline Meat/meat alternatives & 64 & 33.68 & 24 & $12 \cdot 50$ & $<0.001$ & $64 \cdot 17$ & 0.05 & $-0.07,0.18$ \\
\hline Dairy/dairy alternatives & 110 & 57.29 & 71 & $36 \cdot 60$ & $<0.001$ & 54.98 & 0.13 & $0.00,0.26$ \\
\hline Discretionary foods & 9 & 4.64 & 119 & 61.03 & $<0.001$ & $42 \cdot 78$ & 0.04 & $0.00,0.09$ \\
\hline
\end{tabular}

aMcNemar's test.

SFS-ECEC, Short Food Survey-Early Childhood Education and Care.

0.08-1.06 servings across food groups). It is encouraging to note that five of the six food groups were estimated by educators to be within a 0.5 serving of the estimates via direct observation. The largest mean difference between the two methods occurred for breads and cereals. This food group, in particular, may be challenging to estimate due to their inclusion within mixed dishes (e.g. spaghetti bolognaise). Higher reporting of consumption compared to another reference method has been found in other adaptations of the $\mathrm{SFS}^{(28)}$. This finding may be due to a number of reasons. Firstly, due to the nature of the questionnaire, spillage or food sharing that is common in meal times with young children may not adequately be captured by the SFS-ECEC. Secondly, the SFS-ECEC captures a greater proportion of a child's total intake as an additional $15 \cdot 18-25 \cdot 65 \%$ of children were reported to consume breakfast and late snack in care. Consumption during this meal and snack was collected in the recording of usual intake via the SFS-ECEC, but not via direct observations, which was limited to morning tea, lunch and afternoon tea. The consumption of breakfast and late snack was unable to be captured via direct observation due to logistical difficulties and resource constraints. Given the additional recording of breakfast and late snack, a higher reported consumption of the breads and cereals food group in SFS-ECEC might be expected, as these foods are commonly served during these meals (e.g. cereals, crackers). Considering these factors, a relatively higher reported consumption by the SFS-ECEC is not unexpected.

The findings reported here are comparable to those reported by the original SFS, which identified that the SFS reported significantly higher servings of core food groups consumed $(0 \cdot 3-1.5$ servings) compared to 24-h dietary recalls ${ }^{(15)}$. In contrast to the original parentcompleted SFS where discretionary foods were comparatively underestimated, the SFS-ECEC reported higher consumption of discretionary foods. This could reflect differences between educator and parent versions of the SFS where the parents may be more likely to report in a socially desirable manner ${ }^{(10,28)}$.

Low ICCs and wide limits of agreement on the Bland-Altman plots were identified suggesting low levels of agreement between the two methods, and that educator-reported intake of children is likely to vary widely for each individual child. The ICC for fruit and meat/meat alternatives (0.00) suggests there is no agreement, with substantial variation in measures both within and between children. For discretionary foods, an ICC of 0.21 indicates a low level of agreement between the two measures, with slightly less variation within children than between children. The distribution of residual data points in the Bland-Altman plots suggests potential systematic biases in reporting, where the levels of SFS-ECEC reporting are likely higher when mean estimates increase for most food groups. While educators could report children's consumption of foods any number of times per day, week or month, response options for portion sizes consumed were most commonly limited to half, one or two portions, ultimately placing some parameters on the resulting calculation of food group servings consumed. This may somewhat explain the patterns found within the Bland-Altman plots.

In terms of assessing compliance with guidelines for the childcare sector for each food group, the percentage agreement between methods ranged from $42.78 \%$ for discretionary foods to $93.01 \%$ for vegetables. The high percentage agreement for vegetables was due to the high proportion of children who do not meet the guidelines for recommended servings of vegetables. The original SFS was intended to be used to measure diet quality operationalised as adherence to the Australian Dietary Guidelines ${ }^{(15)}$, rather than a measure of food group intake or dichotomous categorisation of guideline compliance. This reflects validation studies where a diet quality index score may be more comparable to the reference method than individual food group serving assessments ${ }^{(15,28)}$. As such, measures specifically designed to capture food group intake may be better suited for adaptation to the ECEC setting using multiple days of direct observation for comparison.

In considering these findings, it is important to recognise that the SFS-ECEC was designed to capture 'habitual or usual intake' over 1 month, while the direct observations were conducted on a single randomly selected day (assuming representation over a usual period). Given the likelihood of variation between usual intake and consumption 
on single day of observations, individual-level agreement findings (e.g. ICCs and Bland-Altman plots) are not unexpected and should be drawn as preliminary. Further, certain food groups that have a typically less consistent pattern of consumption within the ECEC setting (e.g. discretionary foods) may be more vulnerable to issues arising from the assumption that the $1-\mathrm{d}$ snapshot of intake from direct observations is representative of a child's 'usual' intake. The comparison of one dietary assessment method to another method (of known performance and with conceptually different methodology) is common in the validation of dietary measures as there are no absolute methods of usual intake that are free from error ${ }^{(9)}$. The two methods have important differences giving rise to a comparison that encompasses independent error structures. For example, the methods have different frames of reference (i.e. usual intake over 1 month $v$. consumption on a single day), proportion of in-care intake, and differing cognitive demands. However, based on these differences, the findings of the SFS-ECEC compared to direct observations are in the expected direction. A comparison of the SFS-ECEC to multiple days of direct observation may clarify some of the current findings and would allow for comparison of the diet quality component of the tool. While outlier values were excluded from the analysis for both SFS-ECEC and direct observations, a plausible reporter analysis on direct observation data may provide further insights into the variability found between the two methods.

To our knowledge, the SFS-ECEC is the first tool for which acceptability, feasibility and comparison to a known validated measure has been examined to assess the dietary intake of children in care, and was designed to provide an affordable and pragmatic alternative to existing forms of data collection. The tool was modified to allow completion by educators who are responsible for overseeing meal times of children in care. Although a low relative agreement was observed at the individual level, small mean differences in the consumption of core food groups and discretionary foods were observed, suggesting that the tool may be particularly useful in supporting group-level observations. As such, the tool may represent a feasible and acceptable method for public health or community nutritionists to obtain data regarding food group consumption and food choices of children in order to identify food provision modification required to ensure alignment with sector dietary guidelines.

Modifications and refinement of the tool are recommended to increase its overall acceptability, feasibility and to reduce variation in reporting. While low levels of missing responses were found, the administration of the tool via the web or tablet may reduce both the frequency of missed or unquantifiable items and the time taken to complete the tool. Further, given educators reported a high acceptability of the tool, and yet their estimates of consumption were consistently greater than direct observations, modifications may include additional training for educators (e.g. how to estimate food portion sizes within 'mixed' dishes). Amendments to enable the assessment of consumption by individual meals and snacks within SFS-ECEC would likely increase agreement between the two methods. It is noted, however, that such a modification should be weighed against resource intensity and participant burden. Future research to determine the SFSECEC's sensitivity to change is also needed to indicate if this tool can be used to evaluate interventions, in addition to monitoring dietary guideline compliance.

\section{Strengths and limitations}

Strengths of the study include a large sample size ( $>100$ children) adequate for validation studies ${ }^{(29,30)}$; a comprehensively developed tool with input from dietitians, public health nutritionists, health promotion officers with expertise in the childcare setting, child dietary intake measure experts and ECEC service educators; and diligence in selecting educators who would be most appropriate to complete such a measure of child's usual intake in care.

Despite these strengths, results of the study should be considered in light of the following limitations. Firstly, the SFS-ECEC captures child's consumption of all meals while in care, whereas dietary observations only occurred for morning tea, lunch and afternoon tea, which is likely to account for some of the relatively higher reporting identified in the SFS-ECEC. As the SFS-ECEC does not assess intake by meal occasion, this was not accounted for in the analysis. Given that certain food groups may be more likely to be served at certain meal occasions (e.g. breads and cereals at breakfast), an assessment of service menus over the reporting period may have provided further insights into the habitual nature of the meals assessed via direct observations. While a higher food group consumption has been reported in the original SFS tool ${ }^{(15)}$ and in other adaptations of the $\mathrm{SFS}^{(28)}$, a number of potential factors may contribute to the current findings: certain food groups may be more difficult than others to estimate (e.g. those included in mixed meals); the SFS-ECEC does not capture spillages or food sharing that is common among children; and some food groups may not have consistent provision patterns in care (e.g. discretionary foods). Secondly, the SFS-ECEC and direct observations have different assessment periods of the past month and a single day, respectively, resulting in different error structures between the two methods. Despite this, the use of direct observations for comparison provides a commonly used reference standard to understand the performance of the SFS-ECEC. Thirdly, this study was only conducted in services that provided meals to children (i.e. menu services) and, as such, the utility of the tool for services where foods are brought from home is unknown. Fourthly, while participating educators were required to have familiarity with the typical dietary intake of children subject to the SFS-ECEC, 
there was no requirement for educators to be present in the room of the child for the last month, potentially reducing the accuracy of educators reporting for the specified time period. Finally, the study was conducted in services within one state in Australia (NSW), and further validation in other jurisdictions would be beneficial.

\section{Conclusions}

In summary, this quantitative dietary assessment tool has been specifically adapted for use in the ECEC setting and for completion by educators. Study findings indicate the tool to be both highly acceptable and feasible for use in the childcare setting. Study findings also indicate low levels of agreement between the SFS-ECEC compared to direct observations; however, small mean differences in the consumption of core food groups and discretionary foods were observed suggesting that the tool may be useful in estimating group-level intakes. Comparison to an alternate reference method or multiple days of direct observations and some refinements to the tool are recommended to increase comparability of results. The study demonstrates that the SFS-ECEC provides an alternate, feasible, acceptable and low-cost method of assessing the consumption of children in Australian ECEC services, overcoming the common feasibility issues that restrict the assessment of child dietary intake in care.

\section{Acknowledgements}

Acknowledgements: The authors wish to thank participating childcare services, educators, children and data collection staff. Please contact the authors should you like to access the SFS-ECEC. Financial support: This project is funded by the National Health and Medical Research Council (NHMRC) (APP1102943), Cancer Council NSW (PG 16-05), and the Priority Research Centre for Health Behaviour, University of Newcastle. The content of this publication is the responsibility of the authors and do not reflect the views of NHMRC or Cancer Council NSW. Hunter New England Population Health and the University of Newcastle provided infrastructure funding. S.Y. is supported by an Australian Research Council Discovery Early Career Researcher Award. M.F. is supported by Hunter New England Population Health and the Health Research and Translation Centre, Partnerships, Innovation and Research, Hunter New England Local Health District. Conflict of interest: Kirsty Seward is the director of Revive Nutrition Pty Ltd. There are no further conflicts of interest to declare. Authorship: All authors contributed to the conception or design of the work; data acquisition, analysis or interpretation of data and took part in revising the manuscript. All authors give their final approval of this version to be published and agree to be accountable for all aspects of the work. S.Y. and A.F. conceived the study. S.Y., A.F., M.F., R.G., G.H. and T.B. designed the evaluation procedures. R.G. and G.H. developed the Short Food Survey tool, which was adapted for the ECEC setting by S.Y., A.F., M.F., R.G., G.H., T.B. and K.S. A.G. and A.F. led the acquisition of data which was interpreted by A.G., S.Y., A.F., M.F., R.G., G.H. and T.B. A.G. led the drafting of the manuscript; all authors contributed to manuscript drafting. CL conducted statistical analysis. Ethics of buman subject participation: This study was conducted according to the guidelines laid down in the Declaration of Helsinki and all procedures involving study participants were approved by the Hunter New England (16/02/17/4.05) and University of Newcastle (H-20160111) Human Research Ethics Committees. Written informed consent was obtained from all subjects.

\section{Supplementary material}

To view supplementary material for this article, please visit https://doi.org/10.1017/S136898001900404X

\section{References}

1. Bell LK, Golley RK \& Magarey AA (2013) Short tools to assess young children's dietary intake: a systematic review focusing on application to dietary index research. $J$ Obes 2013, 709626.

2. Craigie AM, Lake AA, Kelly SA et al. (2011) Tracking of obesity-related behaviours from childhood to adulthood: a systematic review. Maturitas 70, 266-284.

3. Ward DS, Vaughn A \& Story M (2013) Expert and stakeholder consensus on priorities for obesity prevention research in early care and education settings. Child Obes 9, 116-124.

4. Matwiejczyk L, Mehta K, Scott J et al. (2018) Characteristics of effective interventions promoting healthy eating for preschoolers in childcare settings: an umbrella review. Nutrients 10, 293.

5. Bell LK \& Golley RK (2015) Interventions for improving young children's dietary intake through early childhood settings: a systematic review. Int J Child Health Nutr 4, 14-32.

6. Larson N, Ward DS, Neelon SB et al. (2011) What role can child-care settings play in obesity prevention? A review of the evidence and call for research efforts. J Am Diet Assoc 111, 1343-1362.

7. Yoong SL, Grady A, Wiggers J et al. (2017) A randomised controlled trial of an online menu planning intervention to improve childcare service adherence to dietary guidelines: a study protocol. BMJ Open 7, e017498.

8. Baxter J (2015) Child Care and Early Childhood Education in Australia. Australia: Australian Institute of Family Studies Melbourne.

9. Magarey A, Watson J, Golley RK et al. (2011) Assessing dietary intake in children and adolescents: considerations and recommendations for obesity research. Int $J$ Pediatr Obes 6, 2-11.

10. Livingstone $\mathrm{M} \&$ Robson $\mathrm{P}$ (2000) Measurement of dietary intake in children. Proc Nutr Soc 59, 279-293.

11. Ball SC, Benjamin SE \& Ward DS (2007) Development and reliability of an observation method to assess food intake of young children in child care. J Am Diet Assoc 107, 656-661. 
12. Gubbels JS, Raaijmakers LG, Gerards SM et al. (2014) Dietary intake by Dutch 1-to 3 year-old children at childcare and at home. Nutrients 6, 304-318.

13. Gubbels JS, Gerards SM \& Kremers SP (2015) Use of food practices by childcare staff and the association with dietary intake of children at childcare. Nutrients $\mathbf{7}$, 2161-2175.

14. Golley R, Bell L, Hendrie G et al. (2017) Validity of short food questionnaire items to measure intake in children and adolescents: a systematic review. J Human Nutr Diet 30, 36-50.

15. Hendrie GA, Smith EV \& Golley RK (2014) The reliability and relative validity of a diet index score for 4-11 year-old children derived from a parent-reported short food survey. Public Health Nutr 17, 1486-1497.

16. National Health and Medical Research Council (2013) Australian Dietary Guidelines [NHaMR Council, editor]. Canberra: NHMRC.

17. Centre for Population Health (2014) Caring for Children Birth to 5 Years (Food, Nutrition and Learning Experiences) [NMo Health, editor]. North Sydney: NSW Health.

18. Ball SC, Benjamin SE \& Ward DS (2007) Development and reliability of an observation method to assess food intake of young children in child care. J Am Diet Assoc 107, 656-661.

19. Ball SC, Benjamin Neelon SE \& Ward DS (2014) Diet Observation at Child Care (DOCC): Procedures and Protocol Version 04.29.14. unpublished, provided by authors.
20. Ltd Xyris Software (2017) FoodWorks, 9 ed., vol. Brisbane, Australia: Professional Edition.

21. Lohr KN, Aaronson NK, Alonso J et al. (1996) Evaluating quality-of-life and health status instruments: development of scientific review criteria. Clin Ther 18, 979-992.

22. SAS Institute Inc. (2012) SAS 9.3 Software, 9.3 ed. Cary, NC, USA.

23. Statistics ABo (2018) Socio-Economic Indexes of Australia (SEIFA), 2016. Canberra: Australian Bureau of Statistics.

24. Nelson M (1997) The validation of dietary assessment. Des Concepts Nutr Epidemiol 2, 241-256.

25. Bland JM \& Altman DG (1999) Measuring agreement in method comparison studies. Stat Methods Med Res 8, $135-160$.

26. Landis JR \& Koch GG (1977) The measurement of observer agreement for categorical data. Biometrics 33, 159-174.

27. Burrows T, Collins C, Adam M et al. (2019) Dietary assessment of shared plate eating: a Missing Link. Nutrients 11, 789.

28. Tonkin E, Kennedy D, Golley R et al. (2018) The relative validity of the Menzies Remote Short-Item Dietary Assessment Tool (MRSDAT) in aboriginal Australian children aged 6-36 months. Nutrients 10, 590.

29. Cade J, Thompson R, Burley V et al. (2002) Development, validation and utilisation of food-frequency questionnaires a review. Public Health Nutr 5, 567-587.

30. Peat J, Mellis C \& Williams K (2002) Health Science Research: A Handbook of Quantitative Methods. Thousand Oaks: SAGE. 